\title{
Parathyroid carcinoma
}

Tanweer F, Mani T. United Lincolnshire Hospitals NHS Trust, Lincoln, UK

\section{Abstract:}

Background: Parathyroid carcinoma is a rare condition and its incidence is about 1 in a million ${ }^{1}$. Patients presents with symptom and signs related to primary hyperparathyroidism.

Objective: We report a rare cases of Parathyroid carcinoma treated recently in our department.

Method: Case report and review of the literature concerning the clinical presentation and management.

\section{Results: Patient had one year} history of hypercalcaemia related symptoms. Patient's parathyroid hormone level was very high (141pg/l) along with high serum calcium level (3.48mmol/l). Patient underwent right inferior parathyroidectomy after further biochemical evaluation and localisation scan.

In this case tumour was big $(3 \mathrm{~cm}$ in size, weigh $13.6 \mathrm{gm}$ ) and adherent to recurrent laryngeal nerve. Patient had temporary vocal cord paralysis postoperatively. Patient had biochemical cure.

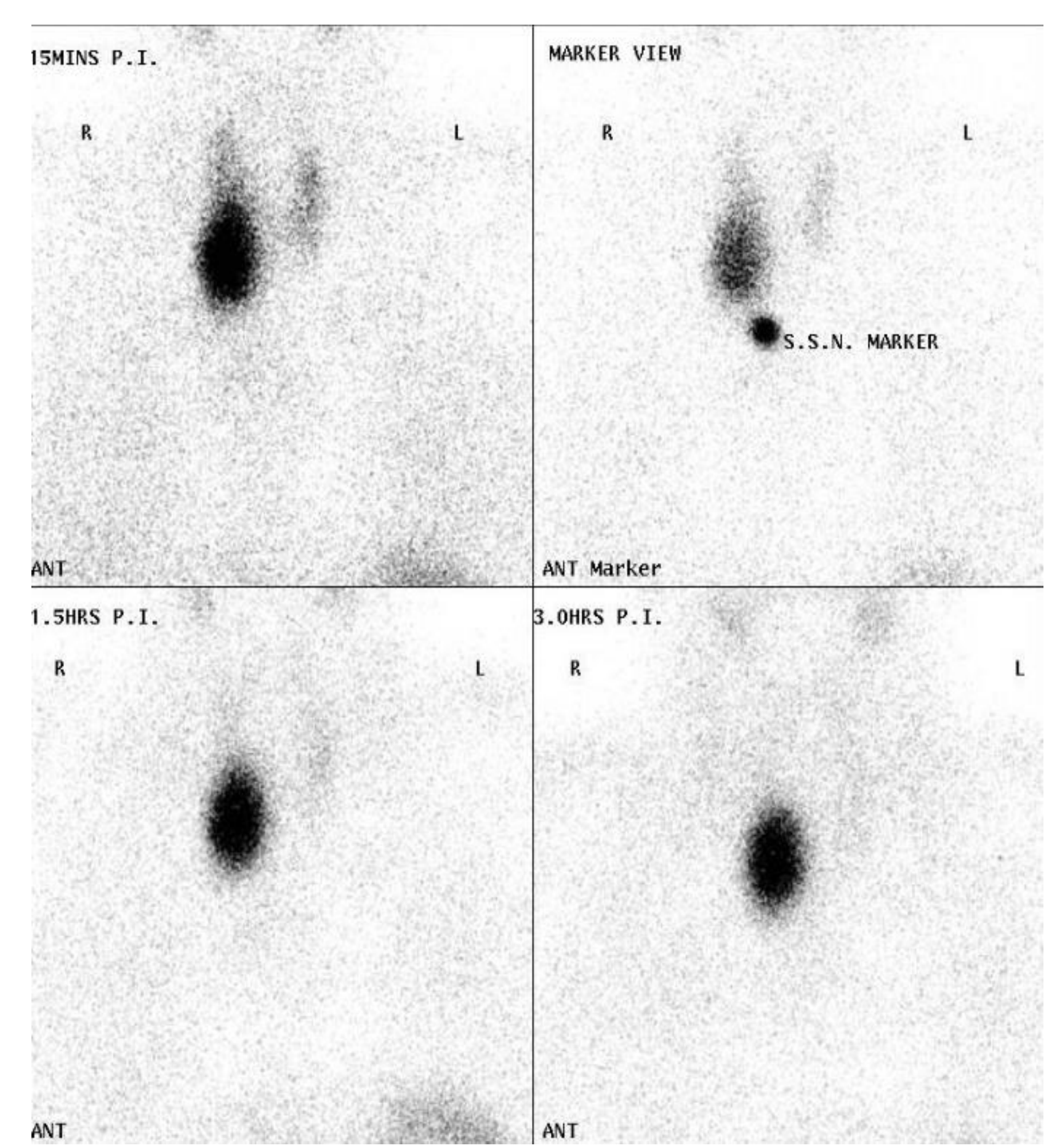

Sestamibi scan

Malignancy was confirmed on full histological examination and confirmation of vascular invasion. ${ }^{2}$ Thyroid MDT suggested for ipsilateral hemithyroidectomy and level ii-iv selective neck dissection to reduce the chance of local recurrence ${ }^{3}$. There were no malignant cells in thyroid or in neck dissection. Post treatment PET scan was also negative.

Conclusion: Parathyroid carcinoma is rare and its management requires team work under the MDT setting.

\section{Reference:}

1.Duan K, Mete Ö.Parathyroid carcinoma: Diagnosis and Clinical Implications. Turk Patoloji Derg. 2015;31 Suppl 1:80-97.

2. Schantz A, Castlenan B. Parathyroid carcinoma. A study of 70 cases. Cancer.1973;31(3):600-5

3. Koea, J.B. and Shaw, J.H. Parathyroid cancer: biology and management. Surg. Oncol. 1999

Nov; 8: 155-165. 